\title{
SPATIAL CLUSTER ANALYSIS OF FEMALE BREAST CANCER DIAGNOSIS IN MISSOURI: USING GIS AND SPATIAL ANALYST FUNCTIONS
}

\author{
Faustine Williams \\ Dr. David O'Brien, Dissertation Supervisor \\ Dr. Stephen Jeanetta, Co-Advisor
}

\begin{abstract}
The stage at cancer diagnosis has a tremendous impact on type of treatment, recovery and survivor. In most cases the earlier the cancer is detected and treated the higher the survival rate for the patient. Various studies have indicated disparities in access to primary care especially access to screening services like mammography for early detection. The purpose of this research was to examine the role of spatial access to health care services on the probability of late detection of female breast cancer diagnosis in Missouri taking into account access and distance to clinics and hospitals. All cancer cases were categorized into two main groups: early defined as in situ and localized stages and late as regional and distant stages. Geographic information system (GIS), spatial analyst functions and logistic regression methods were used to analyze county-level incidence of female breast cancer in Missouri from 2004 to 2008. The GIS results showed that the majority of women in rural Missouri counties do not have access to screening and other health care services. Women had to travel over 60 minutes one way for medical care. This travel burden resulted in a higher probability of late detection. The logistic regression indicated that among younger white and black women, the effect of race and county-level educational score on late detection was similar. For the older group, the effect of race and in particular the lack of education on late detection was greater among blacks than whites. Over all, the age of a woman, race and county-level educational score of residence were the most statistically significant factors in predicting late stage cancer diagnosis among women in Missouri.
\end{abstract}

